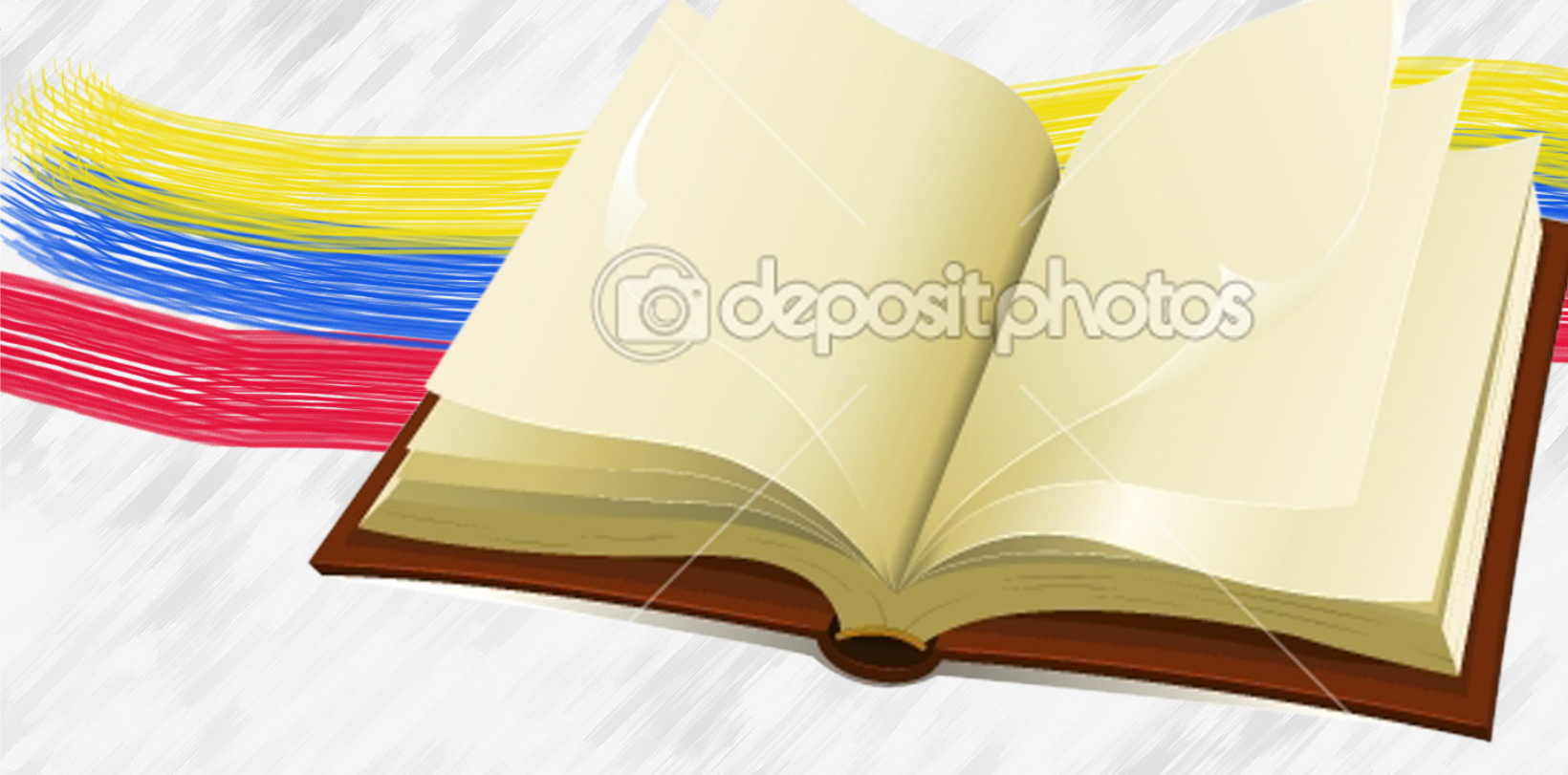

EIDOS, 5

Marzo $\cdot$ Agosto 2012, 53-58

ISSN:1390-499X

elSSN:1390-5007

\title{
EL ESTADO DE LA INVESTIGACIÓN EN LA ENSEÑANZA DE LA LECTURA Y LA ESCRITURA EN LA UNIVERSIDAD COLOMBIANA
}

\section{Murillo ${ }^{1}$, Universidad del Cauca}

Recepción/Received: 2011-11-18

Aceptación/Accepted: 2012-02-15

Publicado/Published: 2012-08-20

Resumen - Este trabajo revisa y estudia la trayectoria investigativa en la enseñanza de la lectura y escritura del ámbito universitario colombiano en la última década. Nos permite de manera general, comprender las líneas de investigación que se trabajan en las universidades en cuanto a la escritura de los géneros propios de las disciplinas y de manera particular, comprender las vías de análisis y los procesos de enseñanza de la escritura que actualmente se llevan a cabo en Colombia. Finalmente y como resultado de la construcción de este estado del arte, logramos detectar cuáles son sus dificultades, cuáles son las contradicciones y las tensiones a fin de iluminarnos en nuevos caminos hacia una didáctica de la lectura y la escritura en la universidad.

Palabras clave - conocimiento, didáctica, disciplinas, enseñanza, escritura, formación, géneros, investigación, lectura, universidad.

Abstract - This paper reviews and analyzes the research trajectory in the teaching of reading and writing at the Colombian university in the last decade. It allows us in general, understand the research that is working in universities, in terms of writing, of gender specific to the field, and in particular, to understand the way of analysis and the teaching of writing, currently carried conducted in Colombia.

Finally, as a result of the construction of this state of the art, we detect what their difficulties, what are the contradictions and tensions in order to illuminate new pathways to teaching reading and writing in the university.

Keywords - Knowledge, didactic, disciplines, teach, write, train, genera, research, read, university.

${ }^{1}$ Mary Edith Murillo Fernández, mmurillo@unicauca.edu.co, Docente de Posgrados, Universidad del Cauca 


\section{INTRODUCCIÓN}

En esta investigación ${ }^{2}$ se concibe que la forma de aprender a escribir en cada disciplina está relacionada con la actividad misma de enseñanza y aprendizaje de los contenidos curriculares de las diferentes materias porque la escritura de los géneros es diferente entre una asignatura y otra; por lo tanto, la enseñanza y el aprendizaje de la escritura es diferente entre un campo disciplinar y otro. Así, cuando los alumnos aprenden una disciplina, lo que aprenden son los géneros de esa disciplina, a pensar, a actuar en esa comunidad discursiva y a integrarse como miembros de ella. El objetivo general es desarrollar una propuesta de enseñanza de los géneros académicos insertos en los aprendizajes de contenidos disciplinares, basada en una intervención pedagógica realizada por la investigadora en una secuencia didáctica en una asignatura del entorno universitario. Para ello, partimos de investigar el estado de la cuestión en lectura y escritura en la universidad. De ahí que esta investigación se constituyó en un punto de partida para las otras investigaciones que realizamos sobre la escritura en la universidad.

\section{Delimitación de la revisión bibliográfica.}

El periodo seleccionado es el último decenio (19992009). A partir de 1999 se establece la Red Colombiana para la Transformación de la Formación Docente en Lenguaje en conexión con la Red Latinoamericana porque marca una pauta importante en las reflexiones sobre la enseñanza de la lengua, ya que su objetivo fue construir una comunidad científica intercultural para generar mecanismos de intercambio, socialización y discusión de trabajos entre docentes e investigadores del país.

En los primeros siete años de esta década, los trabajos sobre la enseñanza de la lectura y la escritura estaban inscritos a eventos generales de currículo, lingüística o semiótica, mientras que en los tres últimos años hay eventos centrados exclusivamente en el análisis de la enseñanza de la lectura y la escritura en la educación superior, para ello se ha convocado El I encuentro de lectura y escritura en la educación universitaria realizado en mayo de 2006 por la Universidad Autónoma de Occidente, y dos Encuentros nacionales sobre políticas institucionales para el desarrollo de la lectura y la escritura en la educación superior, fruto de estos es la Red

${ }^{2}$ Esta investigación se realizó con el apoyo de la Asociación Alban Oficce proyecto No. E06D101690CO y de la Universidad del Cauca. nacional de discusión sobre lectura y escritura en educación superior (Redles) y un proyecto de investigación con las universidades colombianas dirigido por Rincón y Pérez titulado ¿Para qué se lee y se escribe en la universidad colombiana? Un aporte a la consolidación de la cultura académica del país. También en los últimos años las publicaciones se centran en la investigación de la enseñanza y el aprendizaje de la escritura y la lectura en la universidad. En cierta medida, este fenómeno nos permite comprender que la enseñanza de la lengua está inscrita mayoritariamente a los estudios literarios, lingüísticos y semióticos y que paulatinamente se ha ido considerando la didáctica como campo disciplinar que tiene elementos, principios y propósitos particulares para enseñar y aprender los procesos de lectura y escritura en los diferentes entornos académicos. Los trabajos que hemos reseñado en esta investigación mayoritariamente fueron presentados en los eventos académicos tales como seminarios, encuentros, simposios que se realizaron a lo largo de la década seleccionada.

Los criterios de selección para reseñar los libros y los trabajos fueron los siguientes:

- La fecha de publicación: que los trabajos de investigación fueran realizados o publicados durante el periodo comprendido entre 1999 y 2009.

- La temática: que el tema central del corpus reseñado fuera la enseñanza de la lectura y la escritura en la universidad colombiana o latinoamericana.

- El ámbito educativo: que fueran investigaciones o artículos realizados en el ámbito universitario para esta población estudiantil.

- El ámbito latinoamericano y colombiano: que los autores fueran de Colombia y de Latinoamérica o de otra región pero que sus trabajos se refirieran a investigaciones o a experiencias pedagógicas realizadas en el ámbito latinoamericano.

- La relevancia de la investigación: que el trabajo reseñado presente resultados concretos, indicando la importancia y los logros obtenidos de interés e impacto para las instituciones educativas universitarias.

\section{ESTADO DE LA INVESTIGACIÓN EN COLOMBIA}

Después de revisar cada uno de los resúmenes de 132 textos, entre ponencias (105), artículos de revistas y compilaciones (23) y libros (4) y deteniéndonos en la lectura completa de los que consideramos más 
representativos en cuanto al tratamiento del tema: Los procesos de enseñanza y aprendizaje de la escritura en la universidad, podemos agrupar los estudios en Colombia sobre este tema en las siguientes líneas de trabajo:

\section{Los trabajos sobre la formación docente en lectura y escritura.}

Esta línea sobre la formación docente se realiza en dos direcciones, una dirigida a los estudiantes de las licenciaturas, y la otra, a los docentes universitarios en ejercicio. Esta línea se caracteriza por la marcada preocupación en el diseño y evaluación de estrategias metodológicas para lograr la efectividad en los procesos de enseñanza de la lectura y la escritura, las formas metodológicas más usadas son los proyectos de aula y el seminario-taller, ambas con el propósito de que los estudiantes escriban y se apropien de los conceptos desarrollados en el curso a través del debate, la disertación y la socialización de la escritura en la comunidad docente. Se evidencia en las investigaciones que los sujetos de investigación son principalmente los estudiantes de los primeros semestres, para diagnosticar el estado de la lectura y la escritura con el que llegan y para ayudarlos al ingreso de la nueva comunidad académica discursiva. En algunos trabajos el concepto de "buen lector" y la revisión del estado en el que ingresan los estudiantes, a veces tiende a mirarse de manera "remedial" con respecto a los conceptos que no aprendieron en el bachillerato. En los últimos años es notable el sentido epistémico de las propuestas de formación de docentes en los procesos lectores y escritores en relación con los requerimientos que exige el ámbito universitario.

\section{Los trabajos sobre la lectura y la escritura en las disciplinas}

Esta línea se caracteriza por hacer propuestas de intervención pedagógica como una estrategia metodológica para el mejoramiento de los procesos de enseñanza y aprendizaje de la lectura y la escritura, hay un interés por poner en marcha propuestas universitarias que tengan en cuenta el discurso escrito como una actividad cognitiva. Cabe destacar que los trabajos reseñados son investigaciones realizadas generalmente al primer semestre de las carreras ${ }^{3}$, y los tipos discursivos trabajados son preferentemente la argumentación con fines pedagógicos en la enseñanza de conceptos científicos

${ }^{3}$ En Colombia muchas carreras universitarias son semestrales y otras son anuales. Los estudiantes, en su mayoría, reciben cursos de lectura y escritura al ingresar al primer semestre. en disciplinas como las Matemáticas, las Ciencias Naturales, la Medicina, el Deporte y la Fisioterapia. El tipo de género trabajado es esencialmente el resumen y el ensayo argumentativo, aunque también el género autobiográfico se ha trabajado con el objetivo de animar a los estudiantes en la escritura y de revisar las dificultades. Se manifiesta un interés por investigar, conocer y clasificar los géneros más usados en algunas asignaturas y su tratamiento en el aula. Se destacan dos tendencias: 1) trabajos realizados, fundamentalmente, desde la lingüística textual, la semiótica discursiva y la psicología cognitiva, cuyo énfasis ha estado en la lectura y en sus procesos pedagógicos y 2) trabajos realizados a partir del movimiento denominado escribir a través del currículo, nacido en la tradición anglosajona en la década de los 80'. Desde esta otra perspectiva, la escritura tiene una importante función epistémica y como tal, se considera que no debe ser abordada de manera exclusiva en los cursos de lengua. Por el contrario, debe ser trabajada en conexión con los temas que los alumnos estudian y tomando en consideración las formas de escritura propias de las distintas disciplinas.

\section{Políticas institucionales.}

Esta línea de trabajo de políticas institucionales ha estado presente desde los inicios de la década estudiada 1999-2009 como parte constitutiva de una de las mesas de trabajo de la Red colombiana para la transformación de la formación docente. Ahora con La Red nacional de lectura y escritura en la educación superior (REDLEES) creada en noviembre de 2006 en Bogotá y convocada por la Asociación colombiana de universidades (ASCUN), una preocupación central es la política institucional. Esta surge como respuesta a diferentes necesidades manifestadas por la comunidad académica universitaria para propiciar el diálogo, el debate y la construcción de propuestas que faciliten la exploración, la investigación en el campo de la lectura y la escritura en los estudiantes y docentes con el propósito de lograr cambios significativos en la comprensión de la lectura y la escritura como prácticas discursivas necesarias para el desempeño profesional y la construcción del conocimiento. En el II encuentro nacional y I internacional sobre la lectura y la escritura en la educación superior, en Bogotá en septiembre de 2008, se partió de tres ejes sustanciales, uno de ellos sobre políticas institucionales.

Las políticas institucionales son un punto de reflexión en el tema de la enseñanza de la lectura y escritura en 
la universidad. El propósito es formar a los estudiantes en las exigencias discursivas que requiere su ingreso a una disciplina de una comunidad académica y discursiva en particular e integrar al estudiante a su nueva vida universitaria, social y profesional. Se ve una marcada tendencia al estudio del referente lingüístico más que a la construcción de sentido de los géneros discursivos, y aún se ve la orientación de tipo "remedial" que se da a estos cursos en el ámbito universitario para estudiar los contenidos temáticos no vistos en el bachillerato y para crear el placer por la lectura.

\section{Los trabajos sobre la reflexión en la didáctica}

La reflexión en la didáctica como disciplina está presente a lo largo de la década estudiada y se hace más evidente en los últimos trabajos. Al inicio de la década se partió muy tímidamente de esta reflexión ya que los cursos de lectura y escritura y, en general, la enseñanza de la lengua aún estaban inscritos a los estudios lingüísticos y a la crítica literaria más que a la didáctica de la lengua y de la literatura; así que es ya a mediados de esta década que se plantea la necesidad de sistematizar el concepto y el estado de discusión en Colombia como podemos observar en el libro La Didáctica de la lengua materna -Estado de discusión en Colombia constituido por una serie de artículos compilados por Vásquez (2005), producto de un evento para tal fin.

Los trabajos reseñados plantean que los cursos de enseñanza de la lengua se han impartido con el propósito de desarrollar las competencias comunicativas en los estudiantes, partiendo de los conceptos de la Etnografía de la comunicación con la visión de la dimensión sociocultural del ser humano en una comunidad discursiva. Se evidencia que los cursos de enseñanza de lengua en la universidad, de una u otra forma han estado unidos a los estudios lingüísticos, semióticos, literarios bien desde la lingüística textual, o bien con pretensiones al análisis del discurso. Los procesos de lectura y de escritura inicialmente se orientaban desde la lingüística y los estudios literarios, en la actualidad se tiende a orientarlos desde la didáctica de la lengua, teniendo en cuenta la función epistémica.

\section{Trabajos sobre las nuevas tecnologías en la enseñanza de la lectura y la escritura}

En la reflexión sobre cómo enseñar y leer en la universidad es visible que se abordan los interrogantes que suscita el uso de las nuevas tecnologías en el mundo actual.

En los procesos de alfabetización universitaria, las nuevas tecnologías se convierten en un punto de reflexión sobre las implicaciones de las formas de hacer circular la información en los procesos de lectura y de escritura, de ahí que encontremos trabajos de investigación que indaguen sobre los nuevos retos que depara las nuevas tecnologías en relación con los procesos de enseñanza y aprendizaje de la escritura.

\section{CONCLUSIONES}

De esta revisión bibliográfica realizada a lo largo de este capítulo para formular el estado de la investigación en la enseñanza de la lectura y la escritura en la universidad colombiana podemos señalar varias cuestiones:

1. La universidad colombiana en particular cuenta con uno o dos cursos de enseñanza de la lectura y la escritura en los primeros semestres orientados en casi todas las carreras universitarias, estos cursos han sido evaluados y analizados críticamente en investigaciones destinadas para tal propósito. De estos trabajos reseñados se desprenden dos posiciones: una que considera la escritura y la lectura como aprendizajes "acabados", por lo que adjudica a los alumnos, a las condiciones precarias de la sociedad y a los docentes de la secundaria que los estudiantes no sepan escribir en la universidad; otra posición que considera la escritura y la lectura como procesos dinámicos de aprendizaje, en continua evolución, en consecuencia comprende que los estudiantes que llegan a la universidad han ingresado a una nueva comunidad discursiva, por lo cual tendrán que aprender los nuevos géneros discursivos que maneja esta comunidad para aprender los nuevos conocimientos de su programa de estudios universitarios.

De esta situación surge una disyuntiva: enseñar la escritura y la lectura en cursos de carácter "remedial" a fin de "nivelar" a los estudiantes en los conocimientos de la lengua que no aprendieron antes del ingreso a la universidad, o enseñar la escritura y la lectura en cursos de carácter discursivo y dialécticos en los que se considere al estudiante que ingresa a la universidad como un sujeto que trae los conocimientos que necesitaba para secundaria, pero que ahora necesita aprender los géneros propios de la disciplina a la que ha ingresado como 
nuevo miembro de ella.

2. Los cursos de lectura y escritura en la universidad, generalmente son orientados por los docentes del área de lengua quienes conocen muy bien la gramática, la sintaxis de la lengua, los géneros literarios y la crítica literaria, etc., pero como es obvio y natural, desconocen los géneros propios de cada una de las disciplinas del ámbito universitario.

Este hecho ha sido objeto de muchas investigaciones y se expone en los trabajos reseñados de los cuales surge el siguiente interrogante: ¿Quiénes son los docentes más indicados para enseñar los géneros propios de cada disciplina: el profesor de la disciplina o el profesor del área de lengua?

3. En un intento por solucionar esta situación hay propuestas de investigación que se inclinan porque sean los profesores de las disciplinas quienes enseñen los géneros académicos, pues son ellos quienes a través de la escritura, la lectura y la interacción en cada una de sus clases y mientras enseñan los contenidos, harán que sus estudiantes ingresen a la comunidad del conocimiento de un determinado campo del saber científico.

4. Para que lo anterior sea posible, hay trabajos reseñados que proponen crear cursos de lectura y escritura destinados a los profesores universitarios para que ellos participen con sus planteamientos, análisis y disertaciones de los diferentes tipos de géneros discursivos que existen en la escritura de cada disciplina para acceder al conocimiento. De esta manera, se reconoce que los géneros discursivos como actividad social y situada son diferentes unos de otros y que dependen de la situación discursiva, de la pertinencia y del reconocimiento entre los usuarios de una y de otra comunidad para usar unos u otros géneros. Hay otros trabajos reseñados que proponen una intervención pedagógica para desarrollar estrategias cognitivas y metacognitivas en una asignatura con el profesor de la materia y el profesor de lengua.

5. No obstante, la gran mayoría de los trabajos reseñados continúan con propuestas de cursos generales donde se enseña a los estudiantes universitarios las generalidades y los puntos en común que tienen la estructura de tipos de textos como la reseña, el ensayo, el resumen y el comentario, por encontrarlos comunes en el uso de los estudios universitarios. Es visible que en estos cursos generales se enseñan estructuras comunes como la introducción, el desarrollo y las conclusiones, los tipos de elementos de cohesión y coherencia, el manejo de estructuras y superestructuras, pero se dificulta mucho a los docentes y a los estudiantes desligarse de una concepción de escritura para "decir" el conocimiento e ingresar a una concepción de la escritura para construir el conocimiento, porque, si bien es cierto que muchos textos pueden compartir ciertas estructuras comunes, cada disciplina tiene sus especificidades, su situación social y cultural y sus intenciones comunicativas y epistémicas al tener conocimientos específicos.

De esta situación surge una disyuntiva en los procesos de enseñanza de la composición escrita: ¿enseñar cursos generales con temáticas que sean compartidas por numerosas disciplinas como el ensayo argumentativo, la síntesis, la reseña crítica, etc. o cursos de lectura y escritura particulares, que enseñen las especificidades de los géneros discursivos propios de cada disciplina?

\section{BIBLIOGRAFÍA}

Agudelo, W. (2001). Lectura, escuela y medios de comunicación: cambios en la cultura escolar. En Memorias, I Coloquio Internacional y III Regional de la Cátedra UNESCO para la lectura y la escritura en América Latina. Cartagena: CD.ROM.

Arbeláez, O. et al. (2008). La Competencia comunicativa: política institucional en la Universidad Pontificia Bolivariana, Medellín. En Memorias, II encuentro nacional y I internacional sobre la lectura y la escritura en la educación superior. Bogotá: CD.ROM.

Arias, C. (2008). ¿Qué investigan las facultades de educación sobre las didácticas en competencias comunicativas? En Memorias, II encuentro nacional y I internacional sobre la lectura y la escritura en la educación superior. Bogotá: CD-ROM.

[4] Barletta, N. (2001). Intervención metodológica para el desarrollo de las competencias lectoras desde un área particular. En Memorias, I Coloquio Internacional y III Regional de la Cátedra UNESCO para la lectura y la escritura en América Latina. Cartagena: CD-ROM.

] Bentancurt, L y Khon, E. (2001). La didáctica de la lengua en el nivel inicial curso a distancia. En Memorias, I Coloquio Internacional y III Regional de la 
Cátedra UNESCO para la lectura y la escritura en América Latina. Cartagena: CD-ROM.

[6] Cadena, S et al. (2008). Tareas escritas y asignaturas del área profesional. En Narváez y Cadena (Comp.) Los desafíos de la lectura y la escritura en la educación superior: Caminos posibles. Cali: UAO.

[7] Camps, A. (1994). L'ensenyament de la composició escrita. Barcelona: Barcanova.

[8] Cardona, Mª C. et al. (2001). Desarrollo de habilidades cognitivas y apropiación de conceptos científicos a través de la lectura y la escritura, En Memorias, I Coloquio Internacional y III Regional de la Cátedra UNESCO para la lectura y la escritura en América Latina, Cartagena: CD.ROM.

[9] Calderón, A. (2001). La argumentación como estrategia para la elaboración y validación de sentidos matemáticos. En Memorias, I Coloquio Internacional y III Regional de la Cátedra UNESCO para la lectura y la escritura en América Latina. Cartagena: CD-ROM.

[10] Carrasco, A. (2008). Lectura y escritura en la universidad: entre lo que los cursos básicos ofrecen y lo que los estudiantes necesitan. En Memorias, II encuentro nacional y I internacional sobre la lectura y la escritura en la educación superior. Bogotá: CD.ROM.

[11] Chois, P. et al. (2008). Sobre un curso inicial de lectura y escritura: ¿qué y a quiénes Enseñar? En Memorias, II encuentro nacional y I internacional sobre la lectura y la escritura en la educación superior. Bogotá: CD.ROM.

[12] Cisneros, M. (2008). Comprensión lectora y escritora en el momento de ingreso a la educación superior. En Narváez y Cadena (Comp.) Los desafíos de la lectura y la escritura en la educación superior: Caminos posibles. Cali: UAO.

[13] Correa, R. (2005). Didáctica, práctica pedagógica e investigación en el marco de una red de docentes del lenguaje. En Vázquez (Comp.) La Didáctica de la lengua materna -Estado de discusión en Colombia. Bogotá: Editorial Univalle-ICFES.

[14] Corrales, S. y Jiménez, S. (2002). La lectura y la escritura en la universidad: resistencias e insistencias en los procesos de reforma curricular. En Memorias del II coloquio internacional sobre currículo. Popayán: CD.ROM.

[15] Fajardo, M. y Arango, M. (2008). Las funciones de la composición escrita en la formación del docente universitario. En Narváez y Cadena (Comp.) Los desafíos de la lectura y la escritura en la educación superior: Caminos posibles. Cali: UAO.

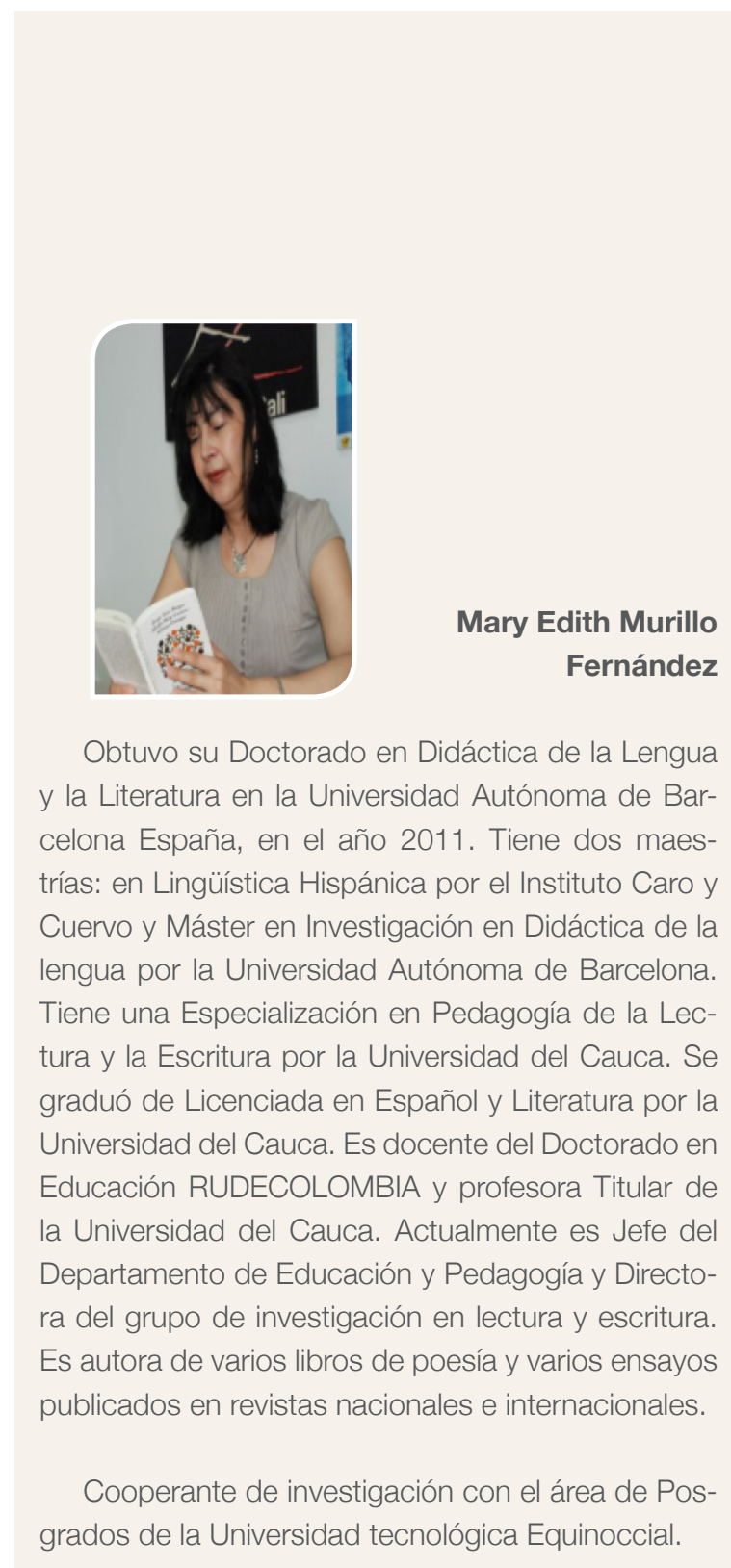

